S. Segawa

Nagoya Math. J.

Vol. 70 (1978), 41-45

\title{
FUNCTIONS MEROMORPHIC ON SOME RIEMANN SURFACES
}

\section{SHIGEO SEGAWA}

Consider an open Riemann surface $R$ and a single-valued meromorphic function $w=f(p)$ defined on $R$. A value $w_{0}$ in the extended complex plane is said to be a cluster value for $w=f(p)$ if there exists a sequence $\left\{p_{n}\right\}$ in $R$ accumulating at the ideal boundary of $R$ such that

$$
\lim _{n \rightarrow \infty} f\left(p_{n}\right)=w_{0} .
$$

The totality of cluster values is referred as the global cluster set for $w=f(p)$ and denoted by $C_{R}(f)$.

In terms of global cluster sets, we define the next two classes of open Riemann surfaces as follows;

Definition. We say that $R \in C_{A B}$ (resp. $C_{H B}$ ) if the global cluster set for each meromorphic function on $R$ is either total or AB-removable (resp. HB-removable).

We have shown in our preceding paper [1] that the following strict inclusion relations hold;

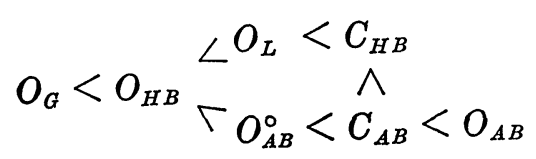

in general, and

$$
O_{G}=C_{H B}<O_{A B}^{\circ} \subset C_{A B}=O_{A B}
$$

for surfaces of finite genus. Moreover we have shown that if there exists a non-constant meromorphic function on $R$ whose global cluster set is $H B$-removable then $R \in O_{G}$. Since $O_{G} \subset C_{H B}$, we deduce the following

Received June 13, 1975.

The author is very grateful to Professor M. Nakai for his helpful suggestions. 
Proposition. In order that $R \in C_{H B}$, it is necessary and sufficient that one of the following alternatives holds:

(i) the global cluster set for any non-constant meromorphic function on $R$ is total;

(ii) there exists a non-constant meromorphic function on $R$ whose global cluster set is HB-removable.

We shall show that the counter-part to the above is valid for $A B$.

THEOREM 1. Let $R$ be an open Riemann surface. Suppose that there exists a non-constant meromorphic function $w=f(p)$ on $R$ whose global cluster set is $A B$-removable. Then $R \in C_{A B}$.

We start by proving the following

LEMMA. Let $R$ be an n-sheeted complete covering surface over the $z$-sphere and $E$ be an AB-removable set in the z-plane. Let $e$ be the set of points on $R$ which lie over $E$. Then $e$ is AB-removable.

Proof. Suppose that $e$ is not $A B$-removable. Since $R$ is closed, there exists a non-constant bounded analytic function $\varphi(p)$ on $R-e$. Without loss of generality we may assume that $\varphi\left(p_{0}\right)=0$ for some $p_{0} \in R$ $-e$, where $p_{0}$ lies over $z_{0} \in \mathscr{C} E$ (throughout this paper, $\mathscr{C}$ denotes the complement). For every $z \in \mathscr{C} E$, let $\left\{p_{1}^{z}, p_{2}^{z}, \cdots, p_{n}^{z}\right\}$ be the set of points of $R$ which lie over $z$. Consider the function

$$
F(z)=\varphi\left(p_{1}^{z}\right) \varphi\left(p_{2}^{z}\right) \cdots \varphi\left(p_{n}^{z}\right) .
$$

Then $F(z)$ is a single-valued bounded analytic function on $\mathscr{C} E$ such that $F\left(z_{0}\right)=0$. On the other hand, since $\varphi(p)$ does not vanish identically, $F(z) \neq 0$ for some $z \in \mathscr{C} E$. Hence $F(z)$ is a non-constant single-valued bounded analytic function on $\mathscr{C} E$. This contradicts the fact that $E$ is $A B$-removable.

Proof of Theorem 1. Since $w=f(p)$ has Iversen's property, $w$ $=f(p)$ covers every point of the $w$-sphere except $C_{R}(f)$ the same finite number of times. We use the same notation $R$ for the covering surface. Let $g(p)$ be an arbitrary non-constant meromorphic function on $R$. We have only to show that $C_{R}(g)$ is $A B$-removable if it is not total. Suppose that $C_{R}(g)$ is not total. Then, without loss of generality, we may assume that $g(p)$ is bounded on a set of points of $R$ which lie over a 
neighbourhood of $C_{R}(f)$.

Choose a point $w_{0}$ in $\mathscr{C} C_{R}(f)$ over which no branch points lie. In a sufficiently small neighbourhood of $w_{0}$, setting

$$
g_{i}(w)=g\left(p_{i}^{w}\right) \quad(i=1,2, \cdots, n),
$$

we obtain $n$ function elements $g_{1}(w), g_{2}(w), \cdots, g_{n}(w)$ with the center at $w_{0}$, where $\left\{p_{1}^{w}, p_{2}^{w}, \cdots, p_{n}^{w}\right\}=f^{-1}(w)$. Set $\mathscr{G}=\left\{g_{1}(w), g_{2}(w), \cdots, g_{n}(w)\right\}$. Here, we notice that every element of $\mathscr{G}$ can be continued everywhere on $\mathscr{C} C_{R}(f)$ by allowing algebraic elements and that some elements of $\mathscr{G}$ may coincide with each other. Hereafter, we partition the proof into two cases.

(i) Suppose that any two elements of $\mathscr{G}$ do not coincide with each other. Consider the symmetric functions of $g_{1}(w), g_{2}(w), \cdots, g_{n}(w)$ :

$$
\begin{aligned}
\sigma_{1}(w) & =\sum_{i} g_{i}(w), \\
\sigma_{2}(w) & =\sum_{i<j} g_{i}(w) g_{j}(w), \\
\vdots & \\
\sigma_{n}(w) & =g_{1}(w) g_{2}(w) \cdots g_{n}(w) .
\end{aligned}
$$

Since any two elements of $\mathscr{G}$ are continued from each other on $\mathscr{C} C_{R}(f)$, each $\sigma_{k}(w)(k=1,2, \cdots, n)$ can be continued everywhere on $\mathscr{C} C_{R}(f)$ and is single-valued meromorphic on $\mathscr{C} C_{R}(f)$. Moreover, by the assumption, each $\sigma_{k}(w)$ is bounded on a neighbourhood of $C_{R}(f)$. Since $C_{R}(f)$ is $A B$ removable, each $\sigma_{k}(w)$ is analytic throughout $C_{R}(f)$ and therefore rational. Hence, all elements of $\mathscr{G}$ are defined by the algebraic function

$$
W^{n}-\sigma_{1}(w) W^{n-1}+\sigma_{2}(w) W^{n-2}+\cdots+(-1)^{n} \sigma_{n}(w)=0 .
$$

Obviously, by the assumption, the above equation is irreducible. Consequently we obtain the $n$-valued algebraic function $G(w)$ defined by the above equation. Denote by $C(G)$ the cluster set for $G(w)$ at $C_{R}(f)$. By the preceding lemma and the fact that $O_{A B}=C_{A B}$ for surfaces of finite genus, we see that $C(G)$ is $A B$-removable. For every cluster value $\zeta$ of $g(p)$, we can find a sequence $\left\{p_{n}\right\}$ in $R$ accumulating at the ideal boundary such that $\lim _{n \rightarrow \infty} g\left(p_{n}\right)=\zeta$ and $w_{n}=f\left(p_{n}\right) \in \mathscr{C} C_{R}(f)$, for any $n$. Since $\left\{w_{n}\right\}$ accumulates to $C_{R}(f), \zeta \in C(G)$, i.e. $C_{R}(g) \subset C(G)$. Therefore the conclusion follows.

(ii) Let $\mathscr{G}^{\sim}=\left\{g_{11}(w), g_{12}(w), \cdots, g_{1 m}(w)\right\}$ be the totality of elements of 
$\mathscr{G}$ which coincide with $g_{1}(w)=g_{11}(w)$ containing $g_{1}(w)$ itself $(m \leq n)$. If there exists no element which does not coincide with $g_{1}(w), g_{1}(w)=\ldots$ $=g_{n}(w)$ is single-valued on $\mathscr{C} C_{R}(f)$. Hence, we have nothing to prove since $O_{A B}=C_{A B}$ for plane regions. Suppose that there exists at least one element $g_{21}(w)$ of $\mathscr{G}$ which does not coincide with $g_{1}(w)$. Choose a path $L$ in $\mathscr{C} C_{R}(f)$ which starts from $w_{0}$ and terminates at $w_{0}$ and along which $g_{21}(w)$ is continued from $g_{11}(w)$. Without loss of generality, we may assume that there exist no branch points of $R$ over $L$. Let $\left\{\lambda_{1}, \lambda_{2}\right.$, $\left.\cdots, \lambda_{m}\right\}$ be the totality of paths on $R$ which lie over $L$ and such that each $\lambda_{i}$ starts from $p_{1 i}^{w_{0}}$, where $p_{1 i}^{w_{0}}$ is the point of $R$ corresponding to the element $g_{1 i}(w)$. Let $p_{2 i}^{w_{0}}$ be the terminal point of $\lambda_{i}$. Then any terminal point does not coincide with the initial point, and does not coincide with any other terminal point. Hence, there exist $m$ function elements $g_{21}(w)$, $g_{22}(w), \cdots, g_{2 m}(w)$ such that each $g_{2 i}(w)$ is continued from $g_{1 i}(w)$ along $\lambda_{i}$ and $g_{21}(w)=g_{22}(w)=\cdots=g_{2 m}(w)$. Consequently, we see that if there exist $l$ distinct elements $g_{11}(w), g_{21}(w), \cdots, g_{l 1}(w)$ in $\mathscr{G}$, then $n=m l$, we can classify $\mathscr{G}$ into the following $m$ classes

$$
\begin{aligned}
\mathscr{G}_{1} & =\left\{g_{11}(w), g_{21}(w), \cdots, g_{l 1}(w)\right\}, \\
\mathscr{G}_{2} & =\left\{g_{12}(w), g_{22}(w), \cdots, g_{l 2}(w)\right\}, \\
& \vdots \\
\mathscr{G}_{m} & =\left\{g_{1 m}(w), g_{2 m}(w), \cdots, g_{l m}(w)\right\},
\end{aligned}
$$

where $g_{11}(w)=g_{12}(w)=\cdots=g_{1 m}(w), g_{21}(w)=g_{22}(w)=\cdots=g_{2 m}(w), \cdots$, $g_{l 1}(w)=g_{l 2}(w)=\cdots=g_{l m}(w)$. Hence, using the same argument as in the first case, we see that every $\mathscr{G}_{i}$ defines the same $l$-valued algebraic function $G(w)$ and that the global cluster set for $G(w)$ at $C_{R}(f)$ is $A B$-removable. Therefore $C_{R}(g)$ is $A B$-removable. This completes the proof.

COROLlaRY 1. In order that $R \in C_{A B}$, it is necessary and sufficient that one of the following alternatives holds:

(i) the global cluster set for any non-constant meromorphic function on $R$ is total;

(ii) there exists a non-constant meromorphic function on $R$ whose global cluster set is AB-removable.

COROLLARY 2. Let $R$ be an n-sheeted complete covering surface over the sphere less an $A B$-removable set. Then $R \in O_{A B}$. 
Proof. By Theorem 1, $R \in C_{A B}$. Hence, $R \in O_{A B}$ since $C_{A B} \subset O_{A B}$.

Related to the preceding theorem, we append the following result

THEOREM $2 *$. Let $R$ be an n-sheeted complete covering surface over the z-sphere less an AB-removable set $E$. Then there exists a meromorphic function on $R$ which is bounded on the set of points of $R$ lying over a neighbourhood of $E$ (or whose global cluster set is not total) and which does not assume identically equal values on any two distinct sheets if and only if $R$ is a subsurface of a closed surface.

Proof. If $R$ is a subsurface of a closed surface, as is well-known, there exists a meromorphic function on $R$ which has a pole only at a non-branch point of $R$ and is regular elsewhere. It is immediate that such a function does not assume identically equal values on any two distinct sheets.

Next, suppose that there exsits a meromorphic function $w=f(p)$ on $R$ satisfying the condition stated above. On $\mathscr{C} E$, we consider the function

$$
F(z)=\left[\prod_{i<j}\left\{f\left(p_{i}^{z}\right)-f\left(p_{j}^{z}\right)\right\}\right]^{2},
$$

where $\left\{p_{1}^{z}, p_{2}^{z}, \cdots, p_{n}^{z}\right\}$ is the set of points of $R$ which lie over $z$. Then we can see that $F(z)$ is single-valued on $\mathscr{C} E$. By the assumption, $F(z)$ is bounded on a neighbourhood of $E$ and does not vanish identically on $\mathscr{C} E$. Since $E$ is $A B$-removable, $F(z)$ is analytic throughout $E$ and hence rational. On the other hand, $F(z)$ vanishes on the points over which branch points lie. Therefore, $R$ has at most finitely many branch points.

\section{REFERENCES}

[1] S. Segawa: On global cluster sets for functions meromorphic on some Riemann surfaces, Nagoya Math. J., 70 (1978), 1-6.

[2] S. Stoïlow: Lecon sur les principes topologiques de la théorie des fonctions analytiques, Gauthier-Villars (1956).

[ 3 ] —-: Sur la théorie topologique des recouvrements Riemanniens, Ann. Acad. Sci. Fenn. A.I. 250/35 (1958).

Department of Mathematics

Daido Inst. of Technology

Daido, Minami, Nagoya 457

Japan

* The author would like to mention his indebtness to the referee who pointed out an important spot in this theorem. 